\title{
Toward Legless Locomotion Control
}

\author{
Ravi Balasubramanian, Alfred A. Rizzi, and Matthew T. Mason \\ The Robotics Institute \\ Carnegie Mellon University. \\ \{bravi, arizzi, matt.mason\}@cs.cmu.edu
}

\begin{abstract}
Motivated by an error-recovery locomotion problem, we propose a control technique for a complex mechanical system by decomposing the system dynamics into a collection of simplified models. The robot considered, The Rocking and Rolling Robot (RRRobot), is a highcentered round-bodied robot that locomotes on a plane by swinging its legs and rocking on its shell. We identify the elements contributing to locomotion through two steps: 1) decoupling the leg-body rotation dynamics from the body-plane contact kinematics, and 2) decoupling the body rotational dynamics into dynamics along each rotational axis. We show, using simulation, that such decoupling provides a good approximation to RRRobot's locomotion and use these models to find an approximate control solution for RRRobot: a mapping between planar translation and leg motions.
\end{abstract}

\section{INTRODUCTION}

With the goal of finding control strategies for locomotion error recovery, this paper focuses on our experience with a six-legged robot called RHex [14]. When RHex becomes high-centered in rugged terrain, its stock locomotion mode fails-RHex's body is stuck on a rock, and its legs do not touch the ground. A high-centered RHex has few options-it can only swing its legs and rock its body. Can we find a structured gait for locomoting a high-centered robot toward recovery?

We study this problem using a prototype highcentered robot called The Rocking and Rolling Robot (RRRobot [1], see Fig. 1), a hemisphere with two short actuated legs. Note that RRRobot's morphology is similar to a two-legged round-bodied high-centered RHex. Earlier, we have shown that oscillatory leg motions induce RRRobot to translate through an interplay of the induced inverted-pendulum-like body rotations and slip-free contact constraints, and we also present gaits that provide linear and curved translation [2]. We call such a locomotion technique legless locomotion (see Fig. 2 for an example gait). This paper provides an understanding of legless locomotion using decoupled models and uses the simplified models to find an approximate control method for RRRobot's planar translation at steady state; that is, we find the leg motions that produce the desired robot translational velocity when the robot settles into its limit (steady state) oscillatory cycle.

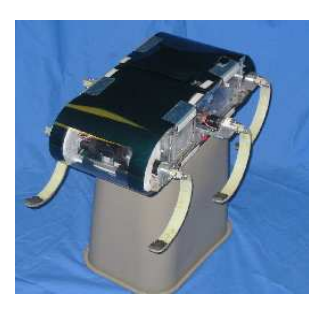

(a)

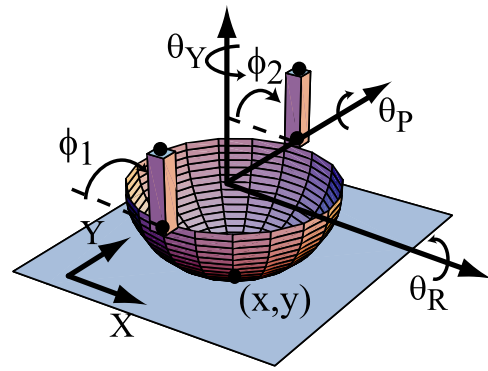

(b)
Fig. 1. (a) A high-centered RHex, and (b) The Rocking and rolling robot.

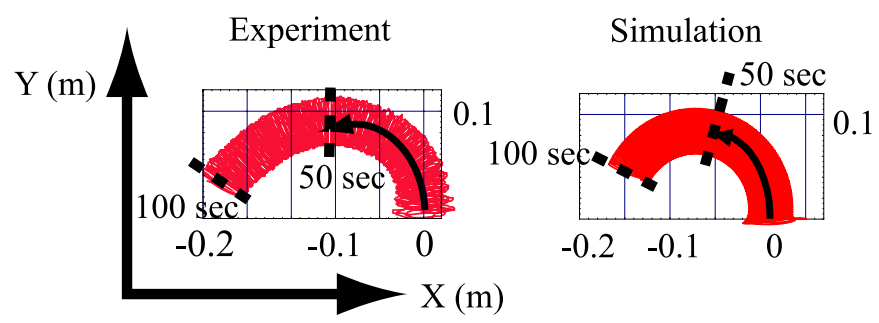

Fig. 2. A legless locomotion gait: counter-clockwise translation produced by body roll-pitch-yaw oscillations resulting from sinusoidal leg motions [2].

Legless locomotion has properties that make control difficult, namely underactuation (two controls and seven degrees of freedom), a configuration-dependent inertia, velocity-related and gravitational drift, and nonholonomic contact constraints. Furthermore, legless locomotion's dynamics structure is difficult to integrate symbolically even for a specific input, thus forcing a numerical analysis.

One way to think about legless locomotion is to consider the contact kinematics and the leg-body dynamics separately (see Fig. 3). For example, considering just the sphere-plane contact kinematics and ignoring the dynamics, interleaved pitch-yaw body rotations produce net displacement [2]. Similarly, considering just the dynamics of leg motions and body rotations ignoring the planar translation, swinging the legs with different phase relationships produces body pitch, roll, and yaw rotations [1]. A key motivation for this paper is to show that such decoupled models provide a good approximation to the original system when recombined. 


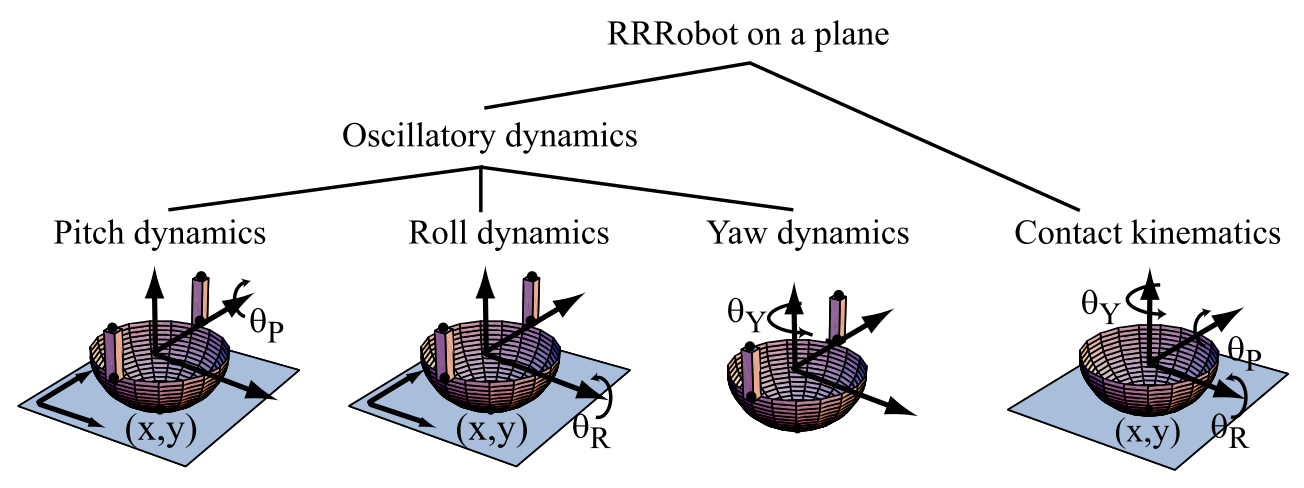

Fig. 3. Decoupled RRRobot dynamics.

\section{RELATED WORK}

We now review related work in three areas: 1) planning techniques for underactuated dynamic systems that locomote using shape changes, 2) dynamics approximation methods, and 3) contact kinematics modeling.

Planning techniques are available for certain types of underactuated dynamic systems that locomote using shape changes, namely systems with no contact constraints and systems with contact constraints but no gravitational drift. The goal in motion planning for systems with no contact constraints is to find the control inputs to reorient the robot. Walsh et al. [9] present a planning strategy to reorient a constant-inertia satellite in space with a varying number of controlled rotors, and Walsh and Sastry [16] extend this approach to a multilinked planar skater. Fernandes et al. [8] provide a nearoptimal planning technique to reorient a falling cat, and Papadopoulos and Dubowsky [6] state that nearly any planning technique used for fixed-base manipulators can be used for planning free-floating space manipulators. The key idea in these approaches is to use angularmomentum conservation principle to find kinematic representations of the dynamic system.

We now look at planning for a system with constant inertia, nonholonomic contact constraints, and no gravitational drift, the Snakeboard [11]. The snakeboard is a variation of the skateboard with controlled wheel directions and a rotor, and it locomotes on a plane through the interplay of stored rotor momentum and varying wheel directions. Bullo and Lewis [7] provide planning primitives for the Snakeboard by finding a kinematic representation, while Shammas et al. [15] provide a natural gait generation strategy using height functions. We note that RRRobot is in a new space of dynamic underactuated systems with a configurationdependent inertia, contact constraints, and gravitational drift. Currently, it is unclear if we can exploit kinematic reductions and height functions for RRRobot control.
Since RRRobot's dynamics are complex, we decouple the dynamics into a set of simplified models. Another technique of simplifying a control system is to linearize its dynamics about an operating point. For example, Laumond [10] gives various linearization techniques to control a nonholonomic car-like robot. But RRRobot's curved translation arises from the non-linearity of its configuration dependent inertia, which is destroyed by linearization. Thus, linearization is not a suitable approach for legless locomotion.

Our assumption of no slip between RRRobot's body and the surface simplifies the modeling and analysis of a high-centered robot's contact, since the relative motion between RRRobot's body and the ground can be expressed as smooth first-order ordinary differential equations [12]. These constraints are functions of the surface curvatures, as derived by Montana [13]. Camicia et al. [4] provide an analysis of the nonholonomic kinematics and dynamics of the Sphericle [3], a hollow ball driven on a planar surface by an unicycle placed inside. The Sphericle and RRRobot have similar contact constraints, but RRRobot's oscillatory locomotion distinguishes its planar motion. We now present models for legless locomotion.

\section{LEGLESS LOCOMOTION MODELS}

In this section, after reviewing RRRobot's dynamics, we derive the simplified models which provide insight into legless locomotion, namely the sphere-plane contact kinematics models and the decoupled rotational dynamics models.

\section{A. RRRobot Dynamics}

RRRobot's locomotion is an interplay of the body attitude oscillations produced by the swinging legs and the no-slip constraints of the rolling sphere. RRRobot's configuration $q_{r}$ consists of the sphere's planar position and orientation $(x, y, R)$ with respect to a global frame and the internal configuration of its legs $\left(\phi_{1}, \phi_{2}\right)$. We represent $R$ using the yaw-pitch-roll body-fixed rotation con- 
vention [5]. Thus, $q_{r}=\left(x, y, R\left(\theta_{R}, \theta_{P}, \theta_{Y}\right), \phi_{1}, \phi_{2}\right)^{T} \in$ $\mathbb{R}^{2} \times S O(3) \times \mathbb{S}^{1} \times \mathbb{S}^{1}$. The equations of motion for RRRobot on a plane take the form

$$
\begin{aligned}
M & \left(q_{r}\right) \ddot{q}_{r}+C\left(q_{r}, \dot{q}_{r}\right) \dot{q}_{r}+G\left(q_{r}\right) \\
& =\tau_{r}+\left(\lambda_{1} \omega^{1}\right)^{T}+\left(\lambda_{2} \omega^{2}\right)^{T}, \\
\omega^{1} \dot{q}_{r} & =0, \\
\omega^{2} \dot{q}_{r} & =0,
\end{aligned}
$$

where $M\left(q_{r}\right) \in \mathbb{R}^{7 \times 7}$ is the positive-definite symmetric non-diagonal configuration-dependent mass matrix, $C\left(q_{r}, \dot{q}_{r}\right) \dot{q}_{r} \in \mathbb{R}^{7}$ is the vector of Coriolis and centrifugal terms, $G\left(q_{r}\right) \in \mathbb{R}^{7}$ is the vector of gravitational terms, and $\tau_{r}=\left(0,0,0,0,0, \tau_{1}, \tau_{2}\right)^{T}$ is the generalized force. The system is underactuated, since we apply input torques $\tau_{1}$ and $\tau_{2}$ to the hip joints only.

The rolling constraints for sphere-plane contact [13] are given by (2) and (3) where $\omega^{1}=\left(1,0,-r \cos \theta_{P} \sin \theta_{Y}, 0,-r \cos \theta_{Y}, 0,0\right)$ and $\omega^{2}=\left(0,1, r \cos \theta_{R} \cos \theta_{Y}, 0,-r \sin \theta_{Y}, 0,0\right)$ are oneforms. The symbols $\lambda_{1}, \lambda_{2} \in \mathbb{R}$ are the magnitudes of the slip-free contact constraint forces in the configuration space. Note that RRRobot's body roll oscillations are small compared to its body pitch oscillations since RRRobot's body pitch axis is directly aligned with its hip joints. See [2] for more details on the dynamics formulation for RRRobot.

For simplicity, we choose leg motions of the form $A \sin (\omega t+\beta)+\gamma$. We set amplitude $A$ to $0.3 \mathrm{rad}$ and frequency $\omega$ to $8 \mathrm{rad} / \mathrm{s}$ and control RRRobot's translation using leg offset and leg phase-difference.

\section{B. Sphere-plane contact kinematics}

In this subsection, we will study sphere-plane contact kinematics [13] independent of the leg-body dynamics. The constraints in (2) and (3) are identical for any sphere rolling on a plane, but RRRobot's inverted pendulum behavior and the associated oscillatory body rotations distinguishes its planar motion. First, since we are specifically interested in RRRobot's net planar motion, we characterize RRRobot's translation speed and direction using its net motion over a cycle and not instantaneous motion. Thus, even if RRRobot's body attitude oscillations cause it to deviate from its average path, we only use its net translation over a cycle to characterize its speed and direction. Second, since RRRobot's contact constraints are non-integrable [13], we do not have an algebraic relationship between net translation and cyclic body rotations.

The sphere-plane contact constraints permit full planar accessibility for oscillatory body rotations. For example, Fig. 4 shows that net contact-point displacement is restricted to the Y-axis for various unit-amplitude
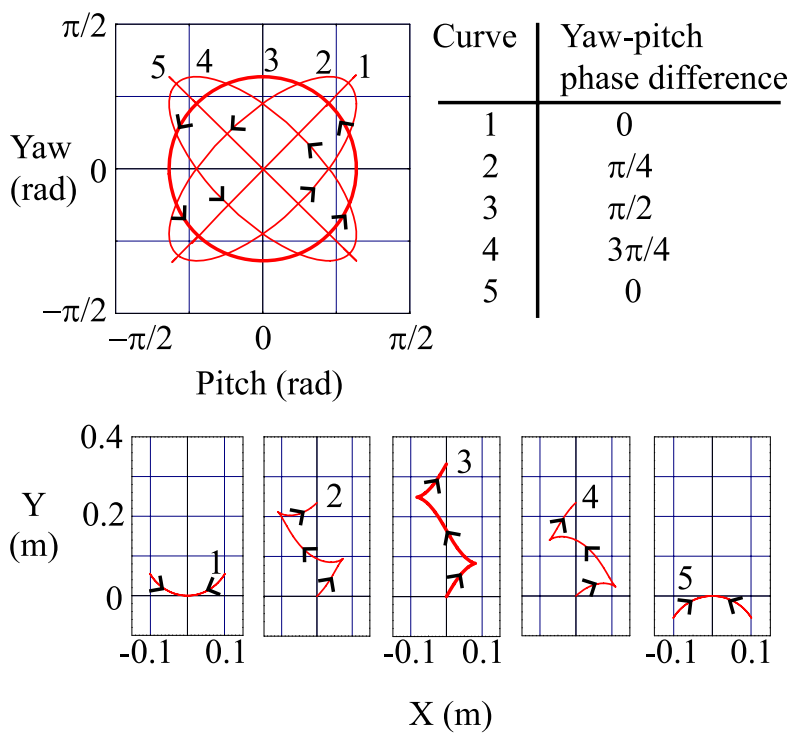

Fig. 4. The path taken by a sphere rolling on a plane changes with body attitude trajectory. The figure shows how the contact point evolves over one cycle for different pitch-yaw phase relationships, given by $\theta_{r}=0, \theta_{p}=\sin (t)$, and $\theta_{y}=\sin \left(t+\beta_{y}\right)$. Sphere radius is $0.12 \mathrm{~m}$

sinusoidal body pitch-yaw phase relationships (with zero roll). A similar relationship exists between net X-axis displacement and various body roll-yaw phase relationships (with zero pitch).

If body roll, pitch, and yaw rotations are non-zero, translation in any direction is possible. Fig. 5 shows translation direction as a function of the phase of sinusoidal body roll and yaw oscillations (with respect to body pitch oscillations). Thus, using different rotational phase and amplitude relationships, we can achieve straight-line displacement along any direction. Furthermore, Fig. 6 provides an example of how translation curves when body yaw drifts in addition to body pitchyaw oscillations. Translation curvature increases with yaw drift rate.

Even though the contact kinematics allow translation in any direction, RRRobot's translation is limited by the body-rotation dynamics. For example, since RRRobot's roll amplitudes are small, its translation is dominated by translation along the body-fixed Y-axis. The limited rollpitch-yaw phase relationships produced by the dynamics also limit translation velocities. However variable curved translation is possible since we can produce net body yaw using out-of-phase leg motions offset from the vertical [1]. This paper focuses on the problem of finding the leg motions to induce body rotations to track a specific path curvature. We now look at some simplified models that provide insight into RRRobot's dynamics. 


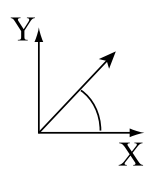

Translation direction

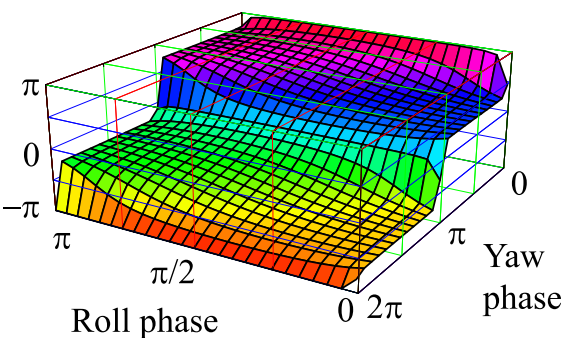

Fig. 5. Translation direction for a sphere (radius $0.12 \mathrm{~m}$ ) on a plane after one cycle in body-rotation space given by $\theta_{r}=\sin \left(t+\beta_{r}\right), \theta_{p}=$ $\sin (t)$, and $\theta_{y}=\sin \left(t+\beta_{y}\right)$.

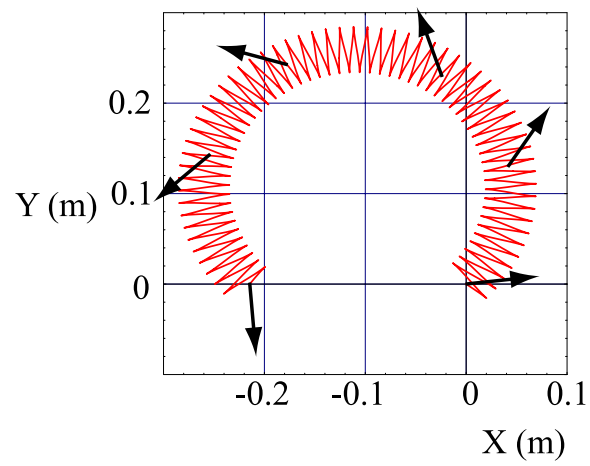

Fig. 6. Contact-point time history for a sphere (radius $0.12 \mathrm{~m}$ ) on a plane starting from the origin for the body-rotation phase relationship given by $\theta_{R}=\theta_{P}=0.15 \sin (8 t)$, and $\theta_{Y}=0.1 t+0.15 \sin (8 t+$ $\pi / 2)$. Arrows indicate robot yaw orientation.

\section{Decoupled Rotational Dynamics}

RRRobot's oscillatory motion embeds two forced inverted pendulums, namely the roll dynamics and the pitch dynamics, whose motion is governed by a combination of gravity and the dynamic effect of leg swing. In contrast, RRRobot's yaw dynamics is produced purely by the dynamic effect of leg swing. These three dynamic systems are coupled through the body-ground rolling contact, complicating analysis.

We propose decoupling the body rotational dynamics and studying the relationship between leg motions and body motions along each axis separately. For example, we create the Pitch model where the body's roll and yaw rotations are disabled. Similarly, the Roll model allows only roll rotations and the Yaw model allows only yaw rotations. Note that the roll and pitch models have a rolling contact, while the yaw model is pivoted. The decoupled oscillatory dynamics models approximate RRRobot's dynamics assuming zero roll-pitch-yaw coupling (see Fig. 3). We will highlight shortly where and why this assumption breaks.

The equations of motion for these fictitious decoupled models take the form

$$
M\left(q_{d}\right) \ddot{q}+C\left(q_{d}, \dot{q}_{d}\right) \dot{q}_{d}+G\left(q_{d}\right)=\tau_{d},
$$

where $q_{d} \in \mathbb{R}^{3}$. The first element of $q_{d}$ is body roll, pitch, or yaw configuration depending on the model, while the last two elements of $q_{d}$ represent leg configuration. The symbols $M\left(q_{d}\right) \in \mathbb{R}^{3 \times 3}, C\left(q_{d}, \dot{q}_{d}\right) \dot{q}_{d} \in \mathbb{R}^{3}, G\left(q_{d}\right) \in \mathbb{R}^{3}$ represent standard mechanical-system terms, and $\tau_{d}=$ $\left(0, \tau_{1}, \tau_{2}\right)^{T}$ is the generalized force. The input torques $\tau_{1}$ and $\tau_{2}$ are applied to the hip-joints, while body rotation is not actuated. We use the contact constraints (2) and (3) to approximately compute RRRobot's translation for the body rotations induced in the decoupled models.

Out-of-phase leg oscillations about the vertical produce body pitch and yaw oscillations about zero, while body roll is negligible. There is a strong match in the induced planar translation paths of the decoupled models and the full dynamics models (see Fig. 7), indicating that body pitch and yaw oscillations are decoupled.

Out-of-phase leg oscillations offset from the vertical produce roll, pitch, and yaw body oscillations. In addition, body pitch tilts from the vertical, and each leg cycle produces net body yaw in both the full-dynamics model and the decoupled dynamics model. The induced planar translation paths compare well (see Fig. 8), but the mapping between the yaw drift rate and various leg trajectories is different for the full-dynamics model and the decoupled models. This is because the yaw inertia in the full dynamics model is a function of body pitch (due to the rolling contact) and leg configuration, while yaw inertia in the decoupled yaw model is only a function of leg configuration (due to the pivot). If we want to use the decoupled dynamics models to approximate RRRobot's motion, some adjustment is required to match the yaw drift in the yaw model and the full dynamics model. In this paper, we vary leg amplitude as a function of leg offset for the decoupled yaw model to make the yaw drift rate match with the full dynamics model. This approximation allow us to approximate RRRobot's translation curvature using the decoupled models.

\section{LEGLESS LOCOMOTION CONTROL}

In this section, we present a technique to control RRRobot like a unicycle with limited turning radius (see Fig. 9). The controls for RRRobot's linear velocity and translation curvature are its leg offset and phase difference (while using sinusoidal leg motions). We show that this control mapping is similar for the decoupled models and the full dynamics models. The key idea is that we can use the decoupled models to predict motion in the full-dynamics model, allowing us to develop advanced control strategies in future.

Figs. 11 and 12 show how translation velocity and curvature depend on leg offset and phase difference for the full model and the decoupled models (using 


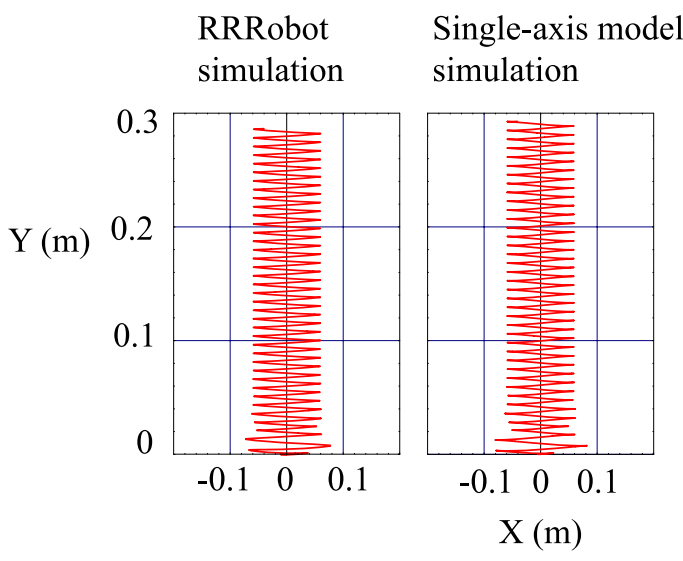

Fig. 7. The lateral translation gait: comparison of RRRobot's motion with motion predicted by the single-axis models over thirty seconds. Leg 1 trajectory: $\pi / 2+0.3 \sin (8 t)$, and leg 2 trajectory: $\pi / 2+$ $0.3 \cos (8 t)$.

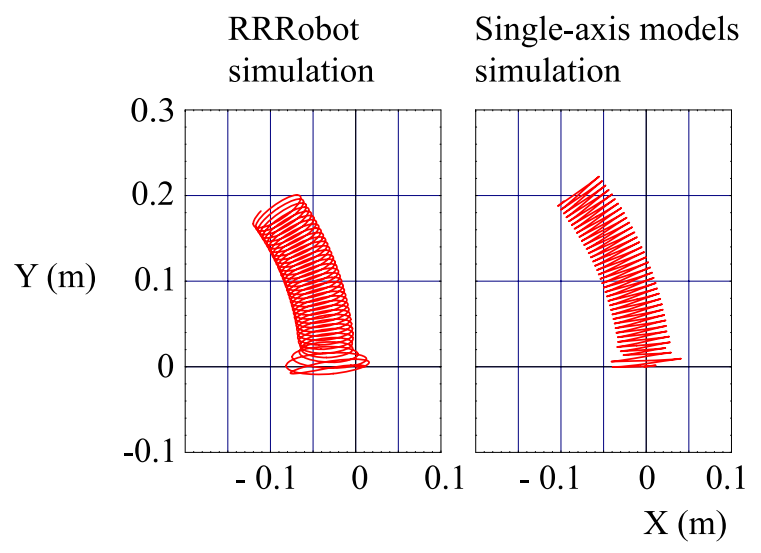

Fig. 8. The circular translation gait: comparison of RRRobot's motion with motion predicted by the single-axis models over thirty seconds. Leg 1 trajectory: $\pi / 4+0.3 \sin (8 t)$, and leg 2 trajectory: $\pi / 4+0.3 \cos (8 t)$.

leg amplitude $0.3 \mathrm{rad}$ (see caveat below), angular frequency $8 \mathrm{rad} / \mathrm{s}$, and measured at the mean of every periodic cycle). The magnitudes and structure of translation curvature match well, but there is a structural difference in the linear-velocity mapping.

This structural difference in the linear-velocity mapping is because of differences in the yaw inertia structure between the full-dynamics model and the simplified dynamics model (see Section III-C). To overcome this difference, we have defined leg amplitude in the decoupled yaw model as a function of leg offset to achieve a good match in curvature control when compared with the full dynamics model. In the decoupled yaw model, the leg motion amplitude is not fixed at 0.3 ; rather it is defined as a function of leg offset (see Fig. 10). Since RRRobot's locomotion is slow in any case, we ignore this small difference in the linear velocity mapping between the decoupled model and the full dynamics model. We use these mappings to derive an inverse relationship for RRRobot control (see Fig. 13).

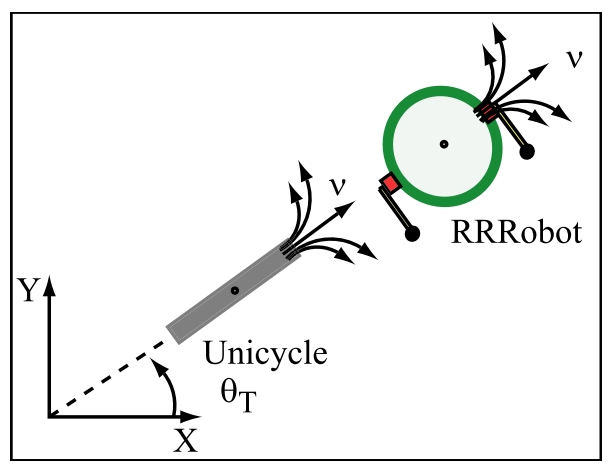

Fig. 9. Similarity in planar translation between a vertical unicycle and RRRobot (top view).

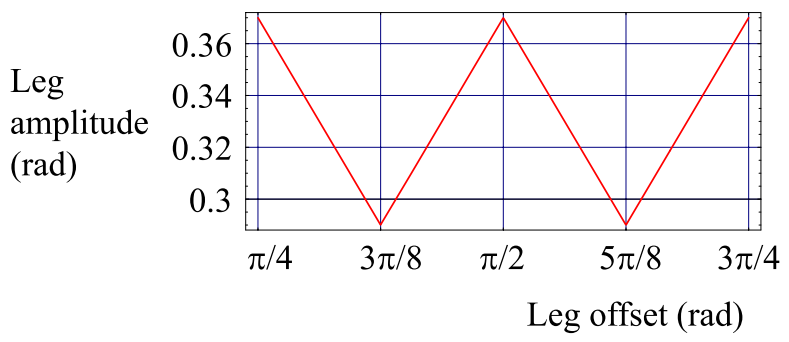

Fig. 10. Amplitude modulation used in the decoupled yaw model.

\section{CONCLUSiON}

This paper provides a geometrical solution to RRRobot control by finding an approximate mapping between legless-locomotion translation and leg trajectories at steady state using dynamics decoupling. Also, future work includes finding a differential mapping between leg trajectory changes and path curvature changes and studying the dynamics of RRRobot without simplifications.

Finally, there are other novel recovery strategies for a high-centered RHex, such as gaits that induce a robot flip over, but this paper focuses only on legless-locomotion control. To improve mobile-robot robustness, we need to find other structured control strategies for situations where conventional locomotion fails.

\section{ACKNOWLEDGMENT}

This work was supported by NSF IIS 0082339, NSF IIS 0222875, and DARPA/ONR N00014-98-1-0747 contracts. We thank Elie Shammas, Amir Shapiro, and Marilena Vendittelli for insightful discussions.

\section{REFERENCES}

[1] R. Balasubramanian, A. A. Rizzi, and M. T. Mason. Legless locomotion for legged robots. In Proceedings of the International Conference on Robots and Intelligent Systems, volume 1, pages 880-885, 2003. 

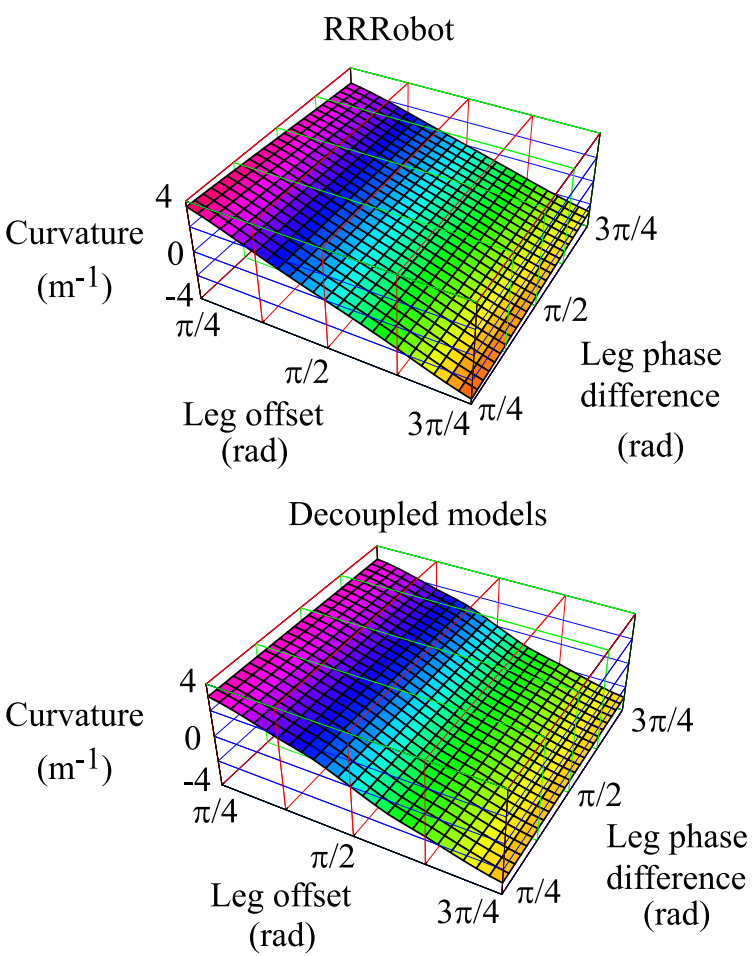

Fig. 11. Comparing RRRobot's dynamics model and its decoupled dynamic models using the mapping between RRRobot's translation curvature and leg trajectories.
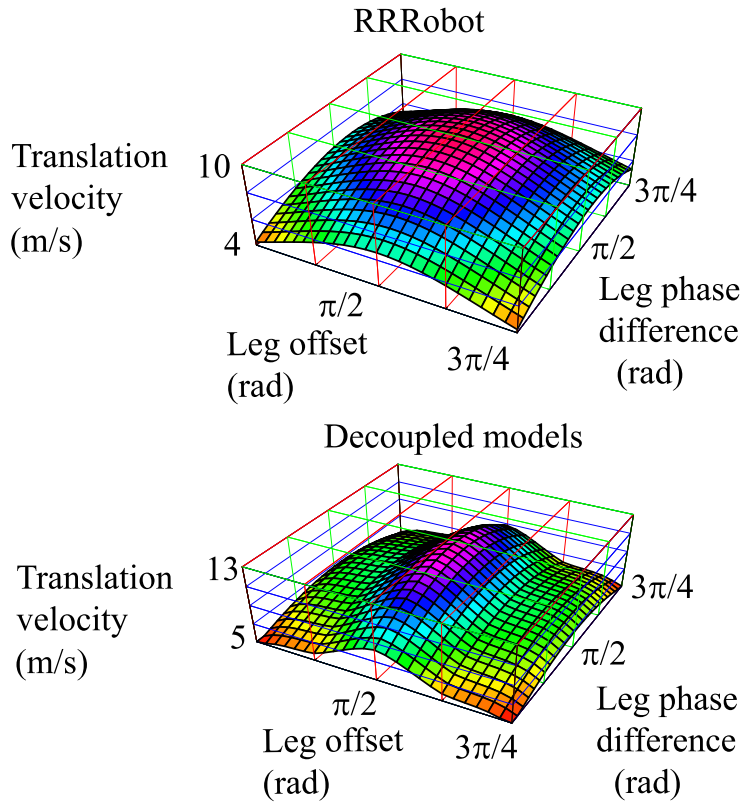

Fig. 12. Comparing RRRobot's dynamics model and its decoupled dynamic models using the mapping between RRRobot's translation velocity and leg trajectories.

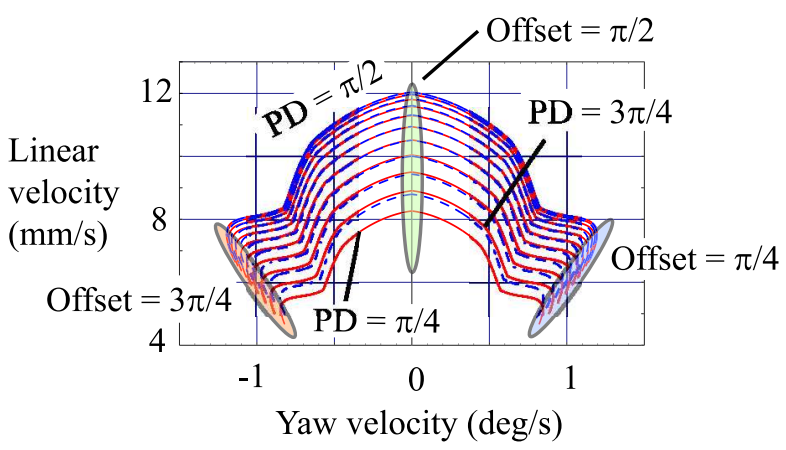

Fig. 13. Mapping between RRRobot linear velocity and yaw velocity and leg offset and leg phase difference (PD).

[2] R. Balasubramanian, A. A. Rizzi, and M. T. Mason. Legless locomotion: Models and experimental demonstration. In Proceedings of the IEEE International Conference on Robotics and Automation, 2004.

[3] A. Bicchi, A. Balluchi, D. Prattichizzo, and A. Gorelli. Introducing the sphericle: an experimental testbed for research and teaching in nonholonomy. In Proceedings of the IEEE International Conference on Robotics and Automation, pages 2620-2625, 1997.

[4] C. Camicia, F. Conticelli, and A. Bicchi. Nonholonomic kinematics and dynamics of the sphericle. In Proceedings of the IEEE International Conference on Intelligent Robots and Systems, pages 805-810, 2000.

[5] J. J. Craig. Introduction to Robotics. Addison Wesley, 1989.

[6] E.Papadopoulous and S.Dubowsky. On the nature of control algorithms for free-floating space manipulators. IEEE Transactions on Robotics and Automation, 7(6):750-758, 1991.

[7] F.Bullo and A.D.Lewis. Kinematic controllability and motion planning for the snakeboard. IEEE Transactions on Robotics and Automation, 19(3):494-498, June 2003.

[8] C. Fernandes, L. Gurvits, and Z. Li. Near optimal nonholonomic motion planning for a system of coupled rigid bodies. IEEE Transactions on Automatic Control, 39(3), 1994.

[9] G.Walsh, A. Sarti, and S. Sastry. Algorithms for steering on the group of rotations. In The proceedings of the American Control Conference, 1993.

[10] J. Laumond. Robot Motion Planning and Control. Springer, 1998.

[11] A. Lewis, J. Ostrowski, R. Murray, and J. Burdick. Nonholonomic mechanics and locomotion: The snakeboard example. In Proceedings of the International Conference on Robotics and Automation, volume 3, pages 2391-2397, 1994.

[12] Z. Li and J. Canny. Motion of two rigid bodies with rolling constraint. IEEE Transactions on Robotics and Automation, 6(1):62-72, Feb. 1990.

[13] D. J. Montana. The kinematics of contact and grasp. The International Journal of Robotics Research, 7(3):17-32, June 1988.

[14] U. Saranli, M. Buehler, and D. E. Koditschek. Rhex: A simple and highly mobile hexapod robot. International Journal of Robotics Research, 20(7):616-631, July 2001.

[15] E. Shammas, H. Choset, and A. Rizzi. Natural gait generation techniques for principally kinematic mechanical systems. In Proceedings of Robotics: Science and Systems, Cambridge, USA, June 2005.

[16] G. C. Walsh and S. S. Sastry. On reorienting linked rigid bodies using internal motions. IEEE Transactions on Robotics and Automation, 11(1), 1995. 